01,05

\title{
Магнитосопротивление и квантовые осцилляции в полуметалле WTe
}

\author{
(C) А.Л. Пирозерский ${ }^{1}$, E.B. Чарная ${ }^{1, \uparrow}$, M.K. Lee Le, $^{2,3}$ L.J. Chang ${ }^{3}$, C.B. Hayмов ${ }^{4}$, \\ А.Н. Доможирова ${ }^{4}$, В.В. Марченков ${ }^{4,5}$ \\ ${ }^{1}$ Санкт-Петербургский государственный университет, \\ Санкт-Петербург, Россия \\ ${ }^{2}$ MOST Instrument Center at NCKU, \\ Tainan, Taiwan \\ ${ }^{3}$ Department of Physics, National Cheng Kung University, \\ Tainan, Taiwan \\ ${ }^{4}$ Институт фризики металлов им. М.Н. Михеева УрО РАН, \\ Екатеринбург, Россия \\ ${ }^{5}$ Уральский фредеральный университет, \\ Екатеринбург, Россия \\ E-mail: e.charnaya@spbu.ru
}

Поступила в Редакцию 21 июля 2021 г.

В окончательной редакции 21 июля 2021 г.

Принята к публикации 23 июля 2021 г.

Обнаружение экстремального магнитосопротивления в немагнитных материалах привлекло внимание к полуметаллу $\mathrm{WTe}_{2}$. Мы провели исследования магнитосопротивления в монокристалле дителлурида вольфрама в диапазоне магнитных полей до $14 \mathrm{~T}$. Магнитосопротивление возрастало с ростом поля по близкому к квадратичному закону без насыщения. Наблюдались осцилляции Шубникова-де Гааза. Найдены четыре фундаментальные частоты в спектре осцилляций, соответствующие двум электронным и двум дырочным карманам, обусловленным сильным спин-орбитальным взаимодействием.

Ключевые слова: полуметалл $\mathrm{WTe}_{2}$, магнитосопротивление, осцилляции Шубникова-де Гааза, спектр осцилляций.

DOI: $10.21883 /$ FTT.2021.12.51662.173

\section{1. Введение}

Слоистые кристаллы дителлурида вольфрама $\mathrm{WTe}_{2}$ являются полуметаллами со структурой $T d$ (ромбическая пространственная группа Pmn $2_{1}$ ) [1], которая остается стабильной при изменении температуры $[2,3]$. Недавно в $\mathrm{WTe}_{2}$, как и в ряде других соединений с металлической проводимостью, был обнаружен экстремальный рост сопротивления при приложении постоянного магнитного поля [4-7]. Величина магнитосопротивления, определяемого как отношение изменения сопротивления к значению сопротивления в нулевом поле, была приблизительно пропорциональна квадрату индукции магнитного поля. Особенностью магнитосопротивления в $\mathrm{WTe}_{2}$ является отсутствие насыщения вплоть до полей $60 \mathrm{~T}$ [4]. На полевой зависимости магнитосопротивления также наблюдались осцилляции Шубникова-де Гааза [8-11]. Спектр осцилляций дает непосредственную информацию об энергии Ферми электронных и дырочных карманов в $\mathrm{WTe}_{2}$. Эти данные представляют особый интерес в связи с тем, что дителлурид вольфрама рассматривается в качестве возможного полуметалла Вейля на основе теоретических предсказаний и экспериментальных результатов, полученных различными методами (см, например, [12-14] и ссылки в этих работах). Измерения магнитосопротив- ления и осцилляций Шубникова-де Гааза при разных температурах позволяют изучать температурную эволюцию электронных и дырочных карманов, что важно при анализе трансформации топологии электронных зон [15]. Имеющиеся в литературе данные о частотах квантовых осцилляций, их температурной зависимости и величине магнитосопротивления не всегда согласуются друг с другом, что возможно связано с различием электронных свойств кристаллов, выращенных в различных условиях и различными методами. В настоящей работе мы представляем результаты исследования магнитосопротивления и осцилляций Шубникова-де Гааза в монокристалле $\mathrm{WTe}_{2}$, выращенном в Институте физики металлов УрО РАН, Екатеринбург.

\section{2. Образцы и эксперимент}

Монокристалл дителлурида вольфрама $\mathrm{WTe}_{2}$ был получен методом химического транспорта. В качестве газообразного реагента использовался бром. Рост кристалла осуществлялся в вакуумированной и запаянной кварцевой ампуле в течение трех недель. Образец для исследований был выколот из выращенного кристалла в форме тонкой пластины толщиной порядка $0.2 \mathrm{~mm}$, ориентированной перпендикулярно оси $c$. Кристаллическая структура, монокристалличность и направления 
кристаллографических осей контролировались методом рентгеновской дифракции. Трехкратные слои атомов $\mathrm{Te}-\mathrm{W}-\mathrm{Te}$ (связь $\mathrm{W}-\mathrm{Te}$ является ковалентной) образуют плоскости, перпендикулярные оси $c$, и связаны между собой слабым взаимодействием Ван-дер-Ваальса. Атомы вольфрама формируют внутри слоев цепочки, ориентированные вдоль кристаллографической оси $a$.

Измерение сопротивления образца в направлении оси $a$ кристалла производилось с помощью системы PPMS-16 производства Quantum Design с использованием встроенной четырехконтактной методики. Температурная зависимость сопротивления в нулевом магнитном поле была получена в диапазоне от 2 до $300 \mathrm{~K}$. Зависимость сопротивления от магнитного поля до 14 Т измерялась при температурах 2 и $5 \mathrm{~K}$. Магнитное поле было направлено вдоль оси $c$ кристалла. В этой геометрии согласно [9] магнитосопротивление имеет наибольшую величину.

\section{3. Результаты и обсуждение}

Изменение сопротивления с температурой в нулевом магнитном поле показано на рис. 1. Увеличение сопротивления с ростом температуры имеет металлический характер. В диапазоне от 90 до $200 \mathrm{~K}$ сопротивление образца демонстрирует линейную зависимость от температуры. Несмотря на сильный рост сопротивления при повышении температуры, отношение $R R R$ значений сопротивлений при $300 \mathrm{~K}$ и при $2 \mathrm{~K}$ равно 31 , что значительно меньше, чем было получено в других работах $[4,8-10]$. Это, по-видимому, связано с несколько большим количеством дефектов в исследуемом нами образце.

Кривые магнитосопротивления MR, измеренные при температурах 2 и $5 \mathrm{~K}$, представлены на рис. 2. Магнитосопротивление в процентах рассчитывалось по формуле

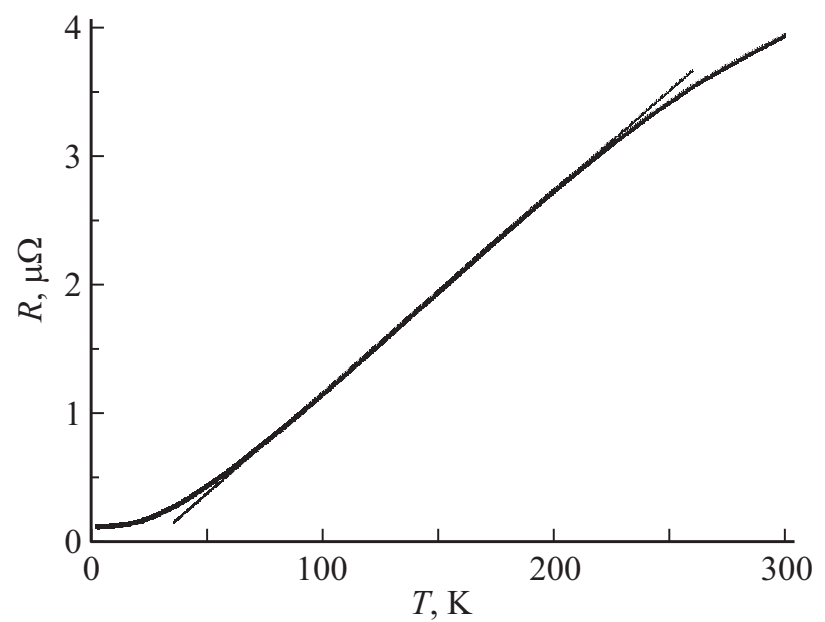

Рис. 1. Температурная зависимость сопротивления в нулевом внешнем магнитном поле. Прямая линия показывает линейную зависимость.

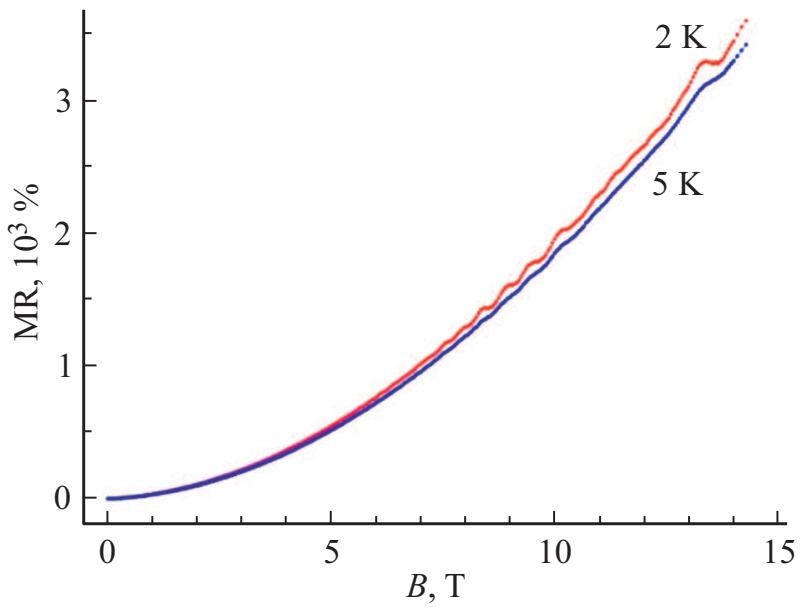

Рис. 2. Зависимость магнитосопротивления от внешнего магнитного поля при температурах 2 и $5 \mathrm{~K}$.

$\mathrm{MR}=[R(B)-R(0)] / R(0) \times 100$, где $R(B)$ и $R(0)$ сопротивление образца в поле $B$ и в нулевом поле соответственно. При $14 \mathrm{~T}$ магнитосопротивление достигает величины $3.45 \cdot 10^{3} \%$ для температуры $2 \mathrm{~K}$ и $3.31 \cdot 10^{3} \%$ для температуры $5 \mathrm{~K}$. Полученные величины магнитосопротивления меньше, чем в работах $[4,8-10]$, что коррелирует с меньшим отношением $R R R$. Как и в предыдущих исследованиях магнитосопротивления в полуметалле $\mathrm{WTe}_{2}$, на зависимости $\mathrm{MR}$ от поля не наблюдается насыщения. Для обеих температур во всем диапазоне полей монотонный рост MR при усилении магнитного поля без учета осцилляций описывается степенной функцией, близкой к квадратичной, $\mathrm{MR} \propto B^{1.78}$. Квадратичная зависимость предсказана теоретически для металлов, полуметаллов и полупроводников в области слабых магнитных полей. Она также наблюдается для компенсированных проводников с замкнутой поверхностью Ферми в области сильных эффективных магнитных полей [16]. Отсутствие насыщения магнитосопротивления свидетельствует о компенсации электронов и дырок $[4,17]$. В рамках двухзонной модели [18], в которой рассматривается одна электронная и одна дырочная зоны, удельное продольное сопротивление в магнитном поле описывается следующим соотношением:

$$
\rho=\frac{1}{e} \frac{\left(n_{e} \mu_{e}+n_{h} \mu_{h}\right)+\left(n_{e} \mu_{e} \mu_{h}^{2}+n_{h} \mu_{h} \mu_{e}^{2}\right) B^{2}}{\left(n_{e} \mu_{e}+n_{h} \mu_{h}\right)^{2}+\left(n_{h}-n_{e}\right)^{2} \mu_{e}^{2} \mu_{h}^{2} B^{2}},
$$

в котором $n_{e}$ и $n_{h}-$ концентрации электронов и дырок, а $\mu_{e}$ и $\mu_{h}-$ соответствующие подвижности. При условии компенсации $n_{e}=n_{h}$, вводя среднюю подвижность $\bar{\mu}$, получаем для магнитосопротивления

$$
\operatorname{MR}=(\bar{\mu} B)^{2} .
$$

Двухзонная модель была использована для объяснения большого магнитосопротивления в висмуте и графите, в которых однако наблюдалось насыщение уже в поле 
Частоты компонент спектра $F$, их относительная интенсивность $I_{r e l}$ и площадь $A$ поперечного сечения поверхности Ферми электронных и дырочных карманов, рассчитанные для 2 и $5 \mathrm{~K}$

\begin{tabular}{c|c|c|c|r|c|c}
\hline & \multicolumn{3}{|c|}{$2 \mathrm{~K}$} & \multicolumn{3}{c}{$5 \mathrm{~K}$} \\
\cline { 2 - 7 } & $F(T)$ & $I_{\text {rel }}$ & $A\left(\mathrm{~nm}^{-2}\right)$ & $F(T)$ & $I_{\text {rel }}$ & $A\left(\mathrm{~nm}^{-2}\right)$ \\
\hline$h_{1}$ & 90.5 & 1 & 0.86 & 88.5 & 0.41 & 0.85 \\
$e_{1}$ & 120.2 & 0.27 & 1.15 & 121.2 & 0.18 & 1.16 \\
$e_{2}$ & 137.3 & 0.49 & 1.31 & 136.8 & 0.20 & 1.30 \\
$h_{2}$ & 158.5 & 0.26 & 1.51 & 158.5 & 0.10 & 1.51 \\
$*$ & 172 & 0.32 & & 173.5 & 0.15 &
\end{tabular}

порядка нескольких Т, что говорит о неполной компенсации электронов и дырок $[19,20]$.

По полученным в настоящей работе данным можно найти среднюю подвижность $\bar{\mu}$. При $2 \mathrm{~K}$ средняя подвижность равна $4.2 \cdot 10^{3} \mathrm{~cm}^{2} \mathrm{~V}^{-1} \mathrm{~s}^{-1}$. Это близко к подвижности, полученной в [21]. Таким образом, в рамках двухзонной модели за большую величину магнитосопротивления отвечает высокая подвижность электронов и дырок при условии равенства их концентраций.

Отметим, что для интерпретации экстремального магнитосопротивления привлекались также модели, учитывающие особенность топологии электронных зон в топологических материалах, приводящую к ограничению обратного рассеяния носителей зарядов [6,22-25]. Однако для кандидата в полуметаллы Вейля $\mathrm{WTe}_{2}$ дополнительные исследования свидетельствуют в пользу справедливости компенсации электронов и дырок [26].

На кривых магнитосопротивления, полученых при 2 и $5 \mathrm{~K}$ (рис. 2), видны квантовые осцилляции Шубниковаде Гааза (ШдГ). Как и следует ожидать, осцилляции ШдГ ослабевают с ростом температуры. Анализ квантовых осцилляций магнитосопротивления проводился с помощью дискретного преобразования Фурье. Для выделения осцилляций на фоне зависимости магнитосопротивления от магнитного поля при обеих температурах монотонный рост магнитосопротивления аппроксимировался полиномом четвертого порядка и вычитался из экспериментальных кривых. На рис. 3 показаны осцилляции ШДГ в зависимости от обратной индукции магнитного поля. Рис. 4 и 5 демонстрируют преобразования Фурье, полученные при температурах 2 и $5 \mathrm{~K}$ соответственно. Наряду с расчетом показаны вклады отдельных компонент спектра и их сумма. Пять частот $F$, соответствующих отдельным компонентам, и интенсивности компонент приведены в таблице. Следуя $[9,10]$ найденные первые четыре частоты следует соотнести с электронным и дырочным карманами, расщепленными за счет спин-орбитального взаимодействия. Этим частотам в спектре осцилляций ШдГ соответствуют в таблице индексы $h_{1}, e_{1}, e_{2}$ и $h_{2}$, где $h$ и $e$ обозначают дырочные и электронные карманы. Цифры 1 и 2 соответствуют двум возможным ориентациям спина. Пятая частота,

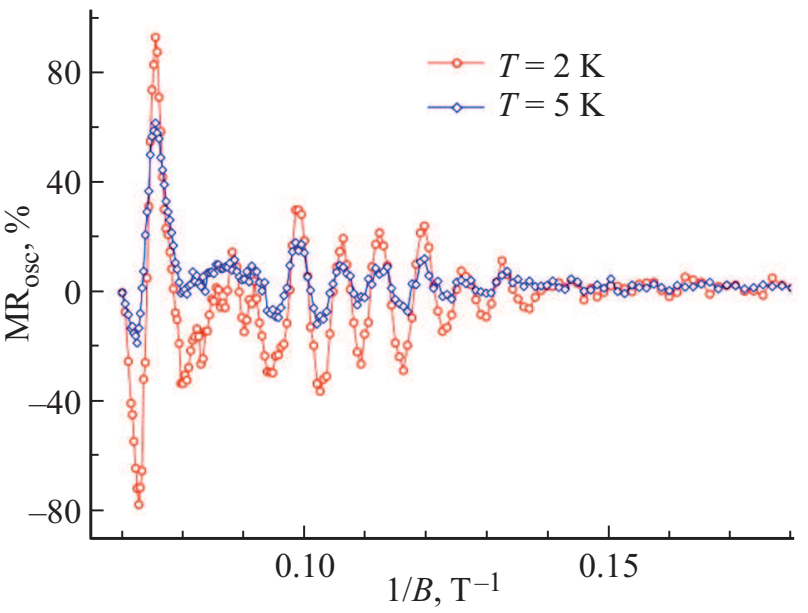

Рис. 3. Осцилляции Шубникова-де Гааза в зависимости от обратной индукции поля при температурах 2 и $5 \mathrm{~K}$.

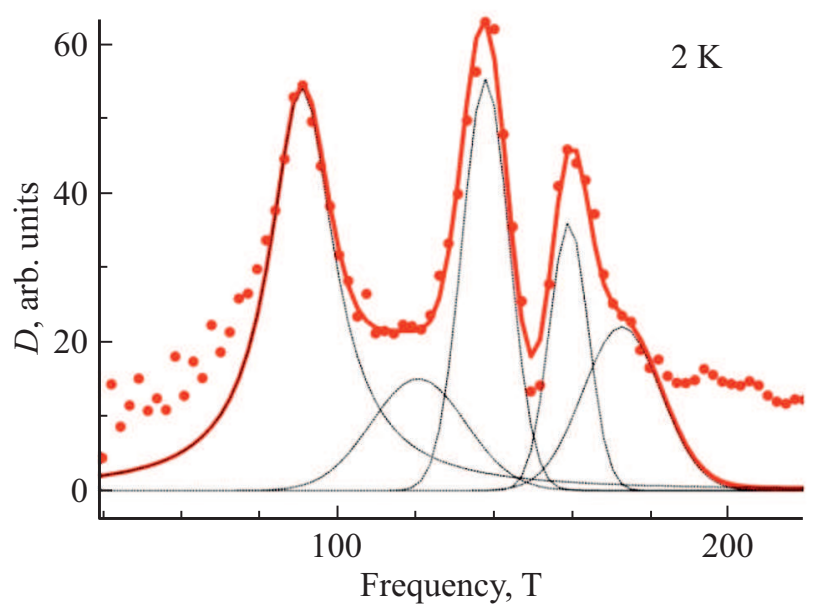

Рис. 4. Спектр осцилляций при температуре $2 \mathrm{~K}$. Точки результат Фурье-пребразования, тонкие сплошные линии рассчитанные отдельные компоненты спектра, толстая сплошная линия - суммарный вклад компонент спектра.

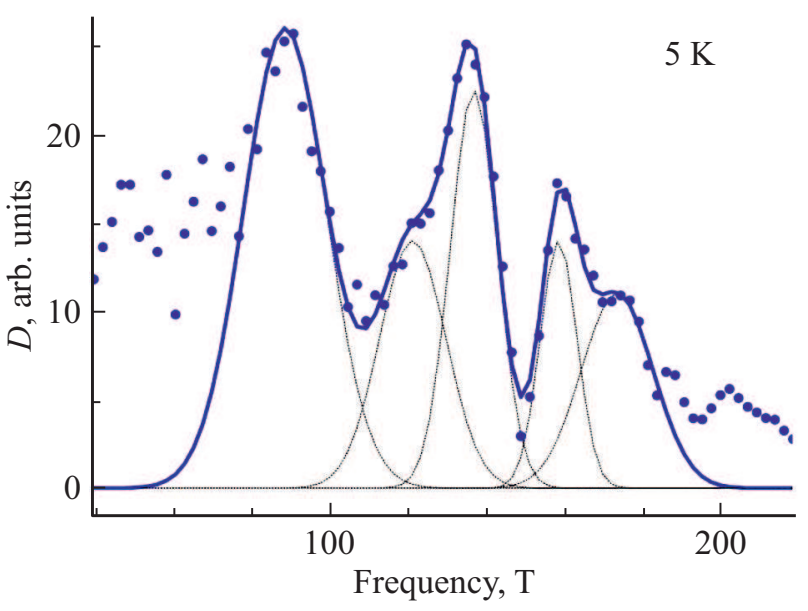

Рис. 5. Спектр осцилляций при температуре 5 K. Точки результат Фурье-пребразования, тонкие сплошные линии рассчитанные отдельные компоненты спектра, толстая сплошная линия - суммарный вклад компонент спектра. 
отмеченная символом *, по-видимому, является второй гармоникой самой низкой частоты. Соотношение Онзагера

$$
F=\left(\Phi_{0} / 2 \pi^{2}\right) A
$$

позволяет по рассчитанным частотам квантовых осцилляций оценить размер $A$ максимального сечения поверхности Ферми электронных и дырочных карманов, перпендикулярного направлению магнитного поля. В (3) $\Phi_{0}$ - квант магнитного потока. Площади сечений также приведены в таблице. Сумма сечений для электронных карманов отличается от суммы сечений для дырочных карманов всего на $3 \%$, что соответствует близкой концентрации электронов и дырок в исследуемом кристалле.

Частоты осцилляций ШдГ, полученные в настоящей работе, можно сравнить с частотами квантовых осцилляций, приведенными в опубликованных исследованиях кристалла $\mathrm{WTe}_{2}[8,9,10,21,27,28]$. В большинстве работ, за исключением [21], было выявлено четыре фундаментальные частоты квантовых осцилляций, разброс значений которых перекрывает различие с полученными нами частотами (таблица). Таким образом, наличие дефектов в исследованном кристалле, которые приводят к снижению $R R R$, не сказывается заметным образом на величине сечения поверхности Ферми электронных и дырочных карманов.

Осцилляции ШдГ могут быть интерпретированы на основе теории Лифшица-Косевича [10]. Согласно этой теории температурная зависимость интенсивности осцилляций позволяет оценить эффективную массу электронов $m^{*}$. Однако, в нашем случае по измерениям при двух температурах оценки для эффективной массы имеют большую погрешность.

\section{4. Заключение}

Исследованный нами монокристаллический образец полуметалла $\mathrm{WTe}_{2}$ характеризуется отношением $R R R=31$, что свидетельствует о присутствии заметного количества дефектов. Магнитосопротивление при температурах 2 и $4 \mathrm{~K}$ достигает величин, превышающих $3 \cdot 10^{3} \%$. Спектр осцилляций Шубникова-де Гааза содержит четыре фундаментальные частоты, согласующиеся с результатами опубликованных исследований магнитосопротивления в дителлуриде вольфрама. Таким образом, дефекты кристаллической структуры, приводящие к снижению магнитосопротивления, не сказываются заметным образом на топологии электронных зон в $\mathrm{WTe}_{2}$.

\section{Финансирование работы}

Исследования финансировались РФФИ, грант 19-57-52001 МНТ-а. Рост кристаллов выполнен в рамках государственного задания МИНОБРНАУКИ России (тема „Спин“, № АAАА-A18-118020290104-2) при частичной поддержке Правительства РФ (постановление № 211, контракт № 02.A03.21.0006).

\section{Конфликт интересов}

Авторы заявляют, что у них нет конфликта интересов.

\section{Список литературы}

[1] B.E. Brown. Acta Cryst. 20, 268 (1966).

[2] D. Kang, Y. Zhou, W. Yi, C. Yang, J. Gou, Y. Shi, S. Zhang, Z. Wang, C. Zhang, S. Jiang, A. Li, K. Yang, Q. Wu, G. Zhang, L. Sun, Z. Zhao. Nature Commun. 6, 7804 (2015).

[3] X.-C. Pan, X. Chen, H. Liu, Y. Feng, Z. Wei, Y. Zhou, Z. Chi, L. Pi, F. Yen, F. Song, X. Wan, Z. Yang, B. Wang, G. Wang, Y. Zhang. Nature Commun. 6, 7805 (2015).

[4] M.N. Ali, J. Xiong, S. Flynn, J. Tao, Q.D. Gibson, L.M. Schoop, T. Liang, N. Haldolaarachchige, M. Hirschberger, N.P. Ong, R.J. Cava. Nature 514, 205 (2014).

[5] E. Mun, H. Ko, G.J. Miller, G.D. Samolyuk, S.L. Bud'ko, P.C. Canfield. Phys. Rev. B 85, 035135 (2012).

[6] T. Liang, Q. Gibson, M.N. Ali, M. Liu, R.J. Cava, N.P. Ong. Nature Mater. 14, 280 (2015).

[7] K. Wang, D. Graf, L. Li, L. Wang, C. Petrovic. Sci. Rep. 4, 7328 (2014).

[8] Z. Zhu, X. Lin, J. Liu, B. Fauqué, Q. Tao, C. Yang, Y. Shi, K. Behnia. Phys. Rev. Lett. 114, 176601 (2015).

[9] D. Rhodes, S. Das, Q.R. Zhang, B. Zeng, N.R. Pradhan, N. Kikugawa, E. Manousakis, L. Balicas. Phys. Rev. B 92, 125152 (2015).

[10] P.L. Cai, J. Hu, L.P. He, J. Pan, X.C. Hong, Z. Zhang, J. Zhang, J. Wei, Z.Q. Mao, S.Y. Li. Phys. Rev. Lett. 115, 057202 (2015).

[11] Y. Wang, K. Wang, J. Reutt-Robey, J. Paglione, M.S. Fuhrer. Phys. Rev. B 93, 121108(R) (2016).

[12] C. Wang, Y. Zhang, J. Huang, S. Nie, G. Liu et al. Phys. Rev. B 94, 241119(R) (2016).

[13] P.K. Das, D.D. Sante, F. Cilento, C. Bigi, D. Kopic et al. Electron. Struct. 1, 014003 (2019).

[14] A.O. Antonenko, E.V. Charnaya, A.L. Pirozerskii, D.Yu. Nefedov, M.K. Lee, L.J. Chang, J. Haase, S.V. Naumov, A.N. Domozhirova, V.V. Marchenkov. Res. Phys. 21, 103793 (2021).

[15] Y. Wu, N.H. Jo, M. Ochi, L. Huang, D. Mou, S.L. Bud'ko, P.C. Canfield, N. Trivedi, R. Arita, A. Kaminski. Phys. Rev. Lett. 115, 166602 (2015).

[16] И.М. Лифшиц, М.Я. Азбель, М.И. Каганов. Электронная теория металлов. Наука, М. (1971).

[17] Y.K. Luo, H. Li, Y.M. Dai, H. Miao, Y.G. Shi, H. Ding, A.J. Taylor, D.A. Yarotski, R.P. Prasankumar, J.D. Thompson. Appl. Phys. Lett. 107, 182411 (2015).

[18] J. Singleton. Band Theory and Electronic Properties of Solids. Oxford University Press, (2001).

[19] P.B. Alers, R.T. Webber. Phys. Rev. 91, 1060 (1953).

[20] Y. Kopelevich, J.H.S. Torres, R.R. da Silva, F. Mrowka, H. Kempa, P. Esquinazi. Phys. Rev. Lett. 90, 156402 (2003).

[21] D. Fu, X. Pan, Z. Bai, F. Fei, G.A. Umana-Membreno, H. Song, X. Wang, B. Wang, F. Song. Nanotechnology 29, 135705 (2018).

[22] H. Weng, C. Fang, Z. Fang, B.A. Bernevig, X. Dai. Phys. Rev. X 5, 011029 (2015). 
[23] Y. Zhao, H. Liu, C. Zhang, H. Wang, J. Wang, Z. Lin, Y. Xing, H. Lu, J. Liu, Y. Wang, S.M. Brombosz, Z. Xiao, S. Jia, X.C. Xie, J. Wang. Phys. Rev. X 5, 031037 (2015).

[24] B.Q. Lv, H.M. Weng, B.B. Fu, X.P. Wang, H. Miao, J. Ma, P. Richard, X.C. Huang, L.X. Zhao, G.F. Chen, Z. Fang, X. Dai, T. Qian, H. Ding. Phys. Rev. X 5, 031013 (2015).

[25] N.J. Ghimire, Y. Luo, M. Neupane, D.J. Williams, E.D. Bauer, F. Ronning. J. Phys.: Condens. Matter 27, 152201 (2015).

[26] Y. Wang, L. Wang, X. Liu, H. Wu, P. Wang, D. Yan, B. Cheng, Y. Shi, K. Watanabe, T. Taniguchi, S.-J. Liang, F. Miao. Nano Lett. 19, 3969 (2019).

[27] K. Tsumura, R. Yano, H. Kashiwaya, M. Koyanagi, S. Masubuchi, T. Machida, H. Namiki, T. Sasagawa, S. Kashiwaya. J. Phys.: Conf. Ser. 969, 012134 (2018).

[28] Y. Luo, H. Li, Y.M. Dai, H. Miao, G. Shi, H. Ding, A.J. Taylor, D.A. Yarotski, R.P. Prasankumar, J.D. Thompson. Appl. Phys. Lett. 107, 182411 (2015).

Редактор К.В. Емиев 